\title{
Phase transition dynamics of single optically trapped aqueous potassium carbonate particles
}

\section{Journal Article}

\section{Author(s):}

Esat, Kıvanç; David, Grégory; Poulkas, Theodoros; Shein, Mikhail; Signorell, Ruth (D)

Publication date:

2018-05-07

\section{Permanent link:}

https://doi.org/10.3929/ethz-b-000268286

Rights / license:

In Copyright - Non-Commercial Use Permitted

Originally published in:

Physical Chemistry Chemical Physics 20(17), https://doi.org/10.1039/c8cp00599k

\section{Funding acknowledgement:}

172472 - Phase Transitions of Ultrafine Aerosol Particles: Condensation, Freezing, and Metal Formation in Confined Systems (SNF) 


\title{
Phase transition dynamics of single optically trapped aqueous potassium carbonate particles ${ }^{\dagger}$
}

\author{
Kıvanç Esat, Grégory David, Theodoros Poulkas, Mikhail Shein, Ruth Signorell*
}

Received Date

Accepted Date

DOI: 10.1039/xxxxxxxxxx

www.rsc.org/journalname
Fast dynamics (down to $10 \mathrm{~ms}$ ) during exposure to changing relative humidity of single optically trapped $\mathrm{K}_{2} \mathrm{CO}_{3}$ particles were observed in the submicron to micron size range with time-resolved broadband light scattering and Raman spectroscopy. The study shows that complex multiple processes accompany efflorescence and deliquescence of unsupported aerosol particles. Efflorescence can occur in a single process in less than $10 \mathrm{~ms}$ (prompt) or proceed via multiple successive processes (multistep) that can last up to more than 10 seconds. The efflorescence relative humidity lies in the range of 9 to $25 \%$. Raman spectra of the effloresced particles reveal that the final state of the particle is independent of the pathway. Deliquescence cycles start with an initial uptake of water followed by multiple complex processes which end at the deliquescence relative humidity $(44-50 \%)$. The study reveals that complex multiple processes during phase transitions are not limited to deposited particles where heterogeneous processes may occur or large particles in the upper micrometer size range as previously observed.

\section{Introduction}

The phase of aerosol particles affects their interaction with the surrounding atmosphere, including their ability to scatter or absorb light. Hence the particles' phase behavior can have an impact on cloud formation ${ }^{1,2}$ and radiative transfer ${ }^{3}$, not only on Earth but also on other planets ${ }^{4}$. The phase behavior of powders is also crucial for applications in food industry ${ }^{5}$ and pharmacy ${ }^{6}$. A major focus of studies on hygroscopic aerosols (mostly inorganic salts) has been measuring the critical relative humidities (RH) at which the liquid particles become solid upon dehydration (efflorescence relative humidity, ERH) and the solid particles become liquid upon hydration (deliquescence relative humidity, DRH). 7,8 This information has given valuable insight into the phase state of aerosols at atmospheric conditions. ${ }^{9,10}$ Nonetheless, such critical relative humidity measurements do not provide information on the dynamics of the particle efflorescence and deliquescence. Recent observations suggest that both phase transitions can be more complex than previously thought and occur in multiple steps with intermediate states. ${ }^{11}$ However, the path and dynamics of the particle phase transitions remain unclear, especially for unsupported aerosol particles smaller than a few millimetres in size. The main objective of this work is to capture the phase transition dynamics of single, optically trapped particles during dehy-

Laboratory of Physical Chemistry, ETH Zürich, Vladimir-Prelog-Weg 2, CH-8093, Zürich, Switzerland. E-mail: rsignorell@ethz.ch

$\dagger$ Electronic Supplementary Information (ESI) available: Bulk solution and single particle Raman scattering measurements. See DOI: 10.1039/b000000x/ dration and rehydration experiments by using time resolved light scattering methods in combination with Raman spectroscopy. Single particle studies avoid potential issues with the interpretation of multistep processes that may arise for particle ensemble studies. Our measurements focus on unsupported, submicron- and micron-sized aerosol particles that are much more relevant to atmospheric processes than millimetre-sized particles.

The phase behavior of unsupported aerosol particles (i.e. isolated in air) can differ from those of particles deposited on substrates. For example, the absence of heterogeneous nucleation sites from the substrates provides access to highly supersaturated metastable states upon drying. The supersaturation of the aerosol particles, which depends on the $\mathrm{RH}$, allows the solute to aggregate to small clusters (nuclei). In the classical picture, the volume Gibbs free energy becomes more dominant than the interfacial free energy if the size of a cluster exceeds a critical value, resulting in spontaneous cluster growth. ${ }^{12-14}$ Classical nucleation theory seems insufficient to explain observations showing more complex pathways that involve multiple steps to form the final most stable crystal form. Such "nonclassical" nucleation pathways have been observed ${ }^{15-19}$ and new theoretical frameworks have been formulated to overcome the limitations of classical nucleation theory ${ }^{11,20-26}$. In addition to multistep processes, multiple (simultaneous) pathways of crystallization in the same system have recently been observed. ${ }^{27,28}$

Deliquescence occurs at a substance-specific DRH, which gives the $\mathrm{RH}$ where the phase transition is complete and which is typically followed by rapid droplet growth. ${ }^{7,8,29,30}$ However, the DRH 
does not provide information about processes at lower RH and earlier stages of the transition. ${ }^{7,20,30,31}$ Wise et al. ${ }^{31}$ showed that $\mathrm{NaCl}$ particles deposited on a substrate are being coated gradually by water before the $\mathrm{RH}$ reaches the deliquescence point. The surface coating was detected through changes in the particle morphology observed prior to deliquescence. ${ }^{32-36}$

To the best of our knowledge, more complex multiple processes during efflorescence and deliquescence have not yet been investigated for unsupported, micron- and submicron-sized aerosol particles, even though unsupported particles in this size range are relevant to atmospheric processes. We have performed dehydration and hydration experiments on single $\mathrm{K}_{2} \mathrm{CO}_{3}$ particles, which were isolated in air with a counter propagating optical tweezer (CPT) inside an environmental cell. Two different types of elastic light scattering, namely two-dimensional angular optical scattering (TAOS) ${ }^{37-39}$ and broadband light scattering spectroscopy (BLS) ${ }^{40-42}$, were used to retrieve information about the particle size, shape (morphology) and refractive index. TAOS measurements are reported in the literature for the study of the phase transitions of micron-sized particles. ${ }^{37,38}$ However, TAOS patterns of submicron-sized particles do not contain sufficient information to detect changes in the particle properties during phase transitions. Ultraviolet BLS, by contrast, has been demonstrated to be an accurate, precise and fast characterization technique for single submicron particles. ${ }^{41}$ Our current setup allows us to study the phase transition dynamics of single particles with a time resolution of $10 \mathrm{~ms}$ and resolve multiple intermediate processes. In addition to elastic scattering, Raman spectroscopy was employed to gain information about the particle's chemical composition via monitoring the $\mathrm{OH}$ band and $\mathrm{CO}_{3}^{2-}$ vibrational modes. The lower sensitivity of single particle Raman spectroscopy limits the timeresolution so that fast intermediate processes cannot be resolved.

\section{Experimental}

Contact free dehydration-hydration of single, submicron- and micron-sized particles was studied in a counter propagating optical tweezer (CPT) using TAOS, BLS and Raman scattering (Fig.1). A continuous wave laser (Laser Quantum, OPUS 532, $532 \mathrm{~nm}$ ) was used to generate the two cross-polarized, counterpropagating Gaussian beams of the CPT (see schematics in 41,43 ). Each beam was focused with a $56.6 \mathrm{~mm}$ working distance, aspherical lens (CVI, BFPL-25.4-50.0-UV) to a beam waist of $\sim 5.6 \mu \mathrm{m}$ in the trapping region. The laser power of each Gaussian beam was around $1 \mathrm{~W}$. Maximum displacements of liquid or solid particles in the trap were less than $50 \mu \mathrm{m}$, which ensured that BLS and Raman measurements could be performed. The stable trapping of efflorescing or deliquescing submicron-sized particle is a challenge, in particular when particles change their shape from spherical to non-spherical upon efflorescence. The advantage of the CPT is that the strong gradient forces reduce particle loss in comparison with other simple optical traps.

Particles were generated by atomizing (TSI 3076 atomizer) a solution of $\mathrm{K}_{2} \mathrm{CO}_{3}$ (Acros $\geq 99 \%$ ) in high purity water (miliQ, $18.2 \mathrm{M} \Omega \mathrm{cm}^{-1}$ ). The generated particle plume was carried into the airtight trapping cell (Fig.1) by a humid $\mathrm{N}_{2}$ flow. A single particle from the plume was trapped, while the remaining parti- cles were flushed out of the cell with a $\mathrm{N}_{2}$ flow of a few seconds duration. The airtight trapping cell allowed us to control the cell atmosphere. The cell temperature was kept at $20 \pm 1{ }^{\circ} \mathrm{C}$ for all measurements. The relative humidity $(\mathrm{RH})$ in the cell was controlled by mixing two gas streams of dry and humidified nitrogen (Pangas, 5.0), respectively, using two flow meters (Bronkhost, EL-FLOW, F-201CV) to control the two streams. The RH was measured every second with a Sensirion SHT31 capacitive sensor ( $\mathrm{RH}$ accuracy $\pm 2 \%$ and temperature accuracy $\pm 0.3^{\circ} \mathrm{C}$ ) that was placed only a few millimeters away from the trapped particle (Fig.1). Measurement of the RH close to the particle is crucial to ensure sufficient accuracy. We have also recorded the $\mathrm{RH}$ at the inlet and outlet of the gas flow into the cell, but these measurements were typically biased by $10 \%$ (e.g. measuring $\mathrm{RH}=60 \%$ instead of $50 \%$ ). The equilibrium relative humidity of saturated aqueous potassium carbonate at room temperature is $43.2 \pm 0.3 \%$ (molality 8.1 mol. $\left.\mathrm{kg}^{-1}\right)^{44}$. Hence, aerosol droplets can become supersaturated at lower RHs. Before the start of each drying experiments (lowering of $\mathrm{RH}$ ), the $\mathrm{RH}$ was kept at a higher value (50-65 \%) than the saturation RH for a sufficiently long time for the droplets to equilibrate.

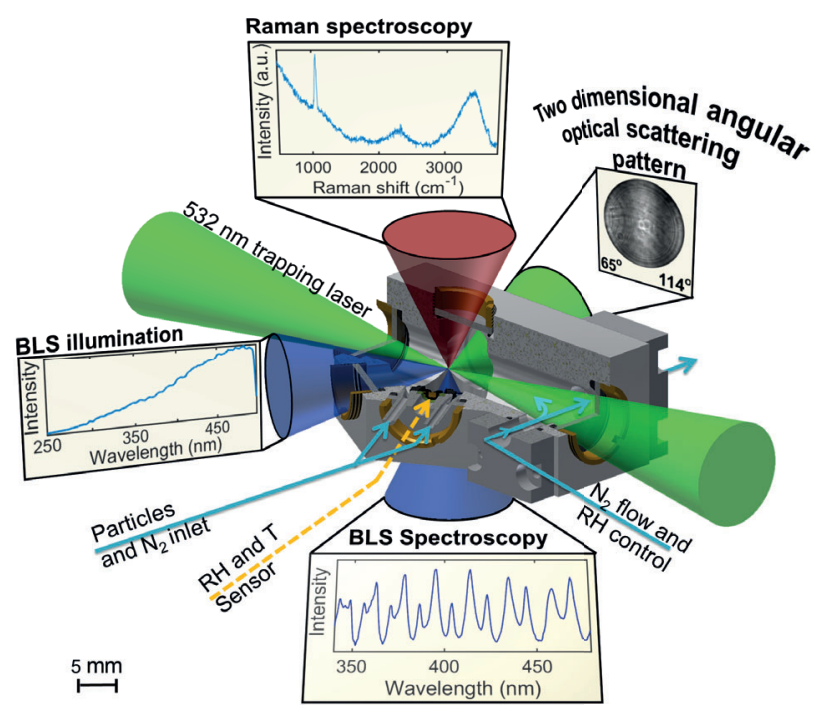

Fig. 1 Scheme of the trapping cell, the counter propagating tweezers (CPT) and the optical methods for single particle characterization. A single aerosol particle is isolated in the center of the trapping cell by the two $532 \mathrm{~nm}$ trapping beams of the CPT. The trapped particle is characterized with broadband light scattering spectroscopy (BLS), two-dimensional angular optical scattering (TAOS) and Raman spectroscopy. The relative humidity $(\mathrm{RH})$ and temperature $(\mathrm{T})$ are measured with a sensor placed a few $\mathrm{mm}$ away from the trapping position.

The principles of the broadband light scattering spectroscopy (BLS) measurements are described in detail in ${ }^{41}$, and are only shortly summarized here. A trapped particle is illuminated by a broadband Xe lamp (Energetiq EQ-99XFC LDLS) and the light scattered by the particle is recorded as a function of the wavelength with a spectrometer (Ocean Optics, Maya2000 PRO with a long pass order filter, $200-1100 \mathrm{~nm}, 25 \mu \mathrm{m}$ slit, $1.1 \mathrm{~nm}$ spectral resolution, down to $10 \mathrm{~ms}$ time resolution). BLS spectra exhibit irregularly spaced peaks with different shape and in- 
tensity (see Fig.1, BLS Spectroscopy). This ripple structure is also referred to as morphology dependent resonances (MDRs) or whispering gallery modes (WGMs). In the present work, we exploit the high sensitivity of the ripple structure (peak positions, shapes and intensities) to particle properties (shape, dimensions and refractive index) as a probe to monitor temporal changes in particle properties during efflorescence and the deliquescence experiments with high time resolution (10 ms). The BLS spectrum of a liquid, spherical particle (before efflorescence or after deliquescence) can further be analyzed by Mie theory, which provides information about the particle radius $R$ and its wavelength dependent refractive index $m(\lambda)$. Best estimates of $R$ and $m(\lambda)$ within their $95 \%$ credible intervals are determined with a global optimizer that compares the whole BLS spectrum (peak positions, intensites and line shape) with model spectra. ${ }^{41,45} \mathrm{~m}(\lambda)$ is represented by two Cauchy parameters, $m_{0}$ and $m_{1}$ (Eq. (1)). $m_{0}$ is the non-dispersive contribution, which is the particle's refractive index at the reference wavelength $\lambda_{0}=400 \mathrm{~nm}\left(m\left(\lambda_{0}\right)=m_{0}\right)$, and $m_{1}$ is the dispersive part. Typical absolute accuracy for particle radii and refractive indices are several $\mathrm{nm}$ and 0.01 , respectively. Note, however, that the precision of relative changes in radius or refractive index are an order of magnitude better. $\mathrm{K}_{2} \mathrm{CO}_{3}$ is nonabsorbing or very weakly absorbing in the wavelength range of interest $(300 \mathrm{~nm}$ to $500 \mathrm{~nm})$ with imaginary parts of the refractive index $(k(\lambda))$ well below $10^{-3}$. The influence of such low $k(\lambda)$ values on the BLS spectra recorded with $1.1 \mathrm{~nm}$ spectral resolution is negligible. ${ }^{41}$

$$
m(\lambda)=m_{0}+m_{1}\left(\lambda_{0}^{2} / \lambda^{2}-1\right)
$$

For a non-spherical particle (e.g. an effloresced particle), the determination of particle shape, dimensions, and refractive indices is not possible from BLS spectra. The retrieval of particle dimensions and refractive indices, for example, would require a priori knowledge of the particle shape, which, however, is not known in the present study (see conclusions). ${ }^{46,47}$ Even though BLS spectra of non-spherical particles cannot provide detailed information on particle properties they still serve as a probe to monitor fast temporal changes in particle properties after efflorescence or before deliquescence.

A microscope objective (Mitutoyo M Plan Apo 20x, numerical aperture $(\mathrm{NA})=0.42$ ) and a CCD camera (Thorlabs DCC1645C) with a maximum sampling rate of $25 \mathrm{~Hz}$ (400 ms) was used for the two-dimensional angular optical scattering pattern (TAOS) measurements. The scattering angle, $\theta$ defines the angle between the incident light (trapping laser) and the direction where the scattering pattern is observed. The described setup can image the scattering pattern within the angular range of $90 \pm 24.8^{\circ}$. The scattering pattern of spherical particles is azimuthally symmetric and hence a regular fringe pattern is observed (see Fig.1, TAOS Pattern). The TAOS of non-spherical particles by contrast is not azimuthally symmetric. Diagnosis of phase transitions with TAOS has been demonstrated for micronsized particles, for example in refs. ${ }^{37,38}$ where the change in the particle's morphology from a liquid to a solid state that accompanies the phase transition was detected through changes in the scattering fringes (spherical shape: regular fringe pattern; nonspherical shape: irregular fringe pattern). However, as will be shown in section 3.2, this method is not applicable to submicronsized particles because the number of fringes and the scattering intensities are typically too low to provide useful information. Hence, in the present work we used TAOS of submicron-sized particles mainly to monitor the particle position in the trap.

The intense trapping laser beams (532 nm) were also used as the excitation source for the Raman spectroscopy. The scattered light was collected with a Mitutoyo M Plan Apo 20x objective and focused onto a fiber of $200 \mu \mathrm{m}$ core size with a second objective (Olympus M-10X, NA $=0.25$ ). The Rayleigh scattering of the excitation laser $(532 \mathrm{~nm})$ is partially filtered with a notch filter (Edmund Optics, 86120, Optical density(OD) 6). To avoid elastic scattering in the range of the Raman measurements, the Xe lamp light was filtered between 500 and $700 \mathrm{~nm}$ with a $500 \mathrm{~nm}$ short pass filter (Edmund optics, 84693, OD 4). The Raman scattering was measured with an Andor spectrometer (SR303iA with Newton camera DU970p-UVB) with a resolution of $0.2 \mathrm{~cm}^{-1}$ using a 600 lines/mm grating (SR3-GRT-0300-0500) and a $10 \mu \mathrm{m}$ slit. The spectrometer was calibrated with a Stellar Inc. SL2 calibration lamp and the Q-branch of gas phase $\mathrm{N}_{2}$ at $2329 \mathrm{~cm}^{-1} .48$ The reproducibility of the peak positions was $\pm 0.5 \mathrm{~cm}^{-1}$. The major limitation of the Raman measurement was the requirement of long exposure times to obtain appropriate signal-to-noise ratios for single particle experiments. Typical exposure times between 1 $\mathrm{s}$ to $60 \mathrm{~s}$ made the observation of very fast processes impossible. The analysis of the Raman spectra concentrates on the symmetric C-O stretching vibration of $\mathrm{CO}_{3}^{2-}\left(v_{1}\left(\mathrm{CO}_{3}^{2-}\right)\right)$ around 2053-2064 $\mathrm{cm}^{-1}$ and on the $\mathrm{O}-\mathrm{H}$ stretching vibration of $\mathrm{H}_{2} \mathrm{O}\left(v_{3}(\mathrm{OH})\right)$ in the region of $2800-3600 \mathrm{~cm}^{-1}$. The peak position of the $v_{1}\left(\mathrm{CO}_{3}^{2-}\right)$ mode is concentration dependent and shifts to lower wavenumbers with increasing salt concentration. ${ }^{49-52}$ Hence, the peak position can be used to determine the particle concentration. For calibration purposes, we performed additional Raman measurements of bulk solutions of different concentration (see ${ }^{\dagger}$ for details), which, however, are limited to the region where the solutions are undersaturated. Isolated particles, by contrast, can easily form supersaturation solutions. While we could record particle Raman spectra in the supersaturated regime, we could not properly calibrate them due to the lack of bulk data. Sometimes the analysis of Raman spectra of spherical particles was complicated by the occurrence of MDRs, which are additional features in the Raman spectra that arise from stimulated Raman scattering. Their positions change with changing droplet radius and concentration (refractive index). While the shape of the sharp $v_{1}\left(\mathrm{CO}_{3}^{2-}\right)$ ) band was hardly affected by MDRs, the shape of the $v_{3}(\mathrm{OH})$ band of $\mathrm{H}_{2} \mathrm{O}$ was more or less strongly distorted by these additional resonance features essentially excluding this band from a quantitative analysis. The spectra of submicron-sized, spherical particles show much fewer and less intense MDRs compared with spectra of micron-sized, spherical particles. Raman spectra of the effloresced particles are not affected by WGMs because of their irregular shape. 


\section{Results}

\subsection{Dehydration-Hydration cycle}

Fig.2 presents a typical dehydration-hydration cycle of a single trapped, aqueous $\mathrm{K}_{2} \mathrm{CO}_{3}$ particle. Panel a shows the temporal change in the $\mathrm{RH}$ while the panels b-g summarize the various results obtained from the BLS and Raman measurements. Individual BLS spectra were measured every $0.5 \mathrm{~s}$ over a total observation time of $250 \mathrm{~min}$. The color-coded scattering intensity of the BLS spectra is shown as a function of wavelength and time in panel b. Yellow, green and blue colors indicate high, medium and low scattering intensity, respectively. The temporal evolution of the particle radius $R$ (panel c) and the non-dispersive (panel d) and dispersive (panel e) contributions $m_{0}$ and $m_{1}$ to the refractive index (Eq. (1)) were extracted from Mie fits to the individual BLS spectra. Panel $\mathrm{f}$ displays the temporal evolution of the $v_{1}\left(\mathrm{CO}_{3}^{2-}\right)$ band recorded in the Raman spectra. The molality in panel $g$ was determined from the position of the $v_{1}\left(\mathrm{CO}_{3}^{2-}\right)$ band before the saturation using a calibration curve (see section 2).

The BLS spectra in Fig.2 b reveal five distinct time windows with a characteristic temporal behavior. The $\mathrm{RH}$ in panel a increases slightly from 60 to $67 \%$ between the initial time $0 \mathrm{~min}$ and $t_{i}=75 \mathrm{~min}$. This results in a slow but continuous drift of the peak positions in the BLS spectra to longer wavelengths (panel b). Panel c shows that this drift comes from a $55 \mathrm{~nm}$ growth of $R$ due to the uptake of water into the droplets caused by the $7 \%$ increase of the RH.

The drying process, which starts at $t_{i}$, leads to evaporation of water from the droplet and to a total decrease in $R$ by $\sim 250 \mathrm{~nm}$ up to the time denoted $t_{E}$ when the particle efflorescence sets in. Consistently, the particle refractive index at $400 \mathrm{~nm}$ increases over time (panel d) because the salt concentration (panel g) increases steadily. Note that in the wavelength region that is of interest here the refractive index of pure water is lower than that of solid $\mathrm{K}_{2} \mathrm{CO}_{3}$. In panel g, we only provide values for molalities up to the bulk saturation point $\left(8.1 \pm 0.7 \mathrm{~mol} \mathrm{~kg}^{-1}\right.$ at $\left.43 \% \mathrm{RH}\right)$ because of the lack of proper calibration data for the $v_{1}\left(\mathrm{CO}_{3}^{2-}\right)$ band position. Wavelength dependent refractive indices (panels $\mathrm{d}_{\text {and }} \mathrm{e}^{\dagger}$ ) and Raman peak positions (panel $\mathrm{f}^{\dagger}$ ), by contrast, are reported over the whole saturation regime. To the best of our knowledge, this is the first time that such data in the supersaturated regime are reported for aqueous $\mathrm{K}_{2} \mathrm{CO}_{3}$ solutions. The peak positions in the BLS spectra (panel b) show slow and more or less continuous drifts to shorter wavelengths in the time window from $t_{i}$ to $t_{E}$.

This behavior changes completely at $t_{E}$ when an abrupt change in the BLS peak positions/intensities is observable that is faster than our time resolution. We identify the onset of the efflorescence by the first abrupt changes in the BLS spectra upon drying and denote the $\mathrm{RH}$ at this point as the efflorescence relative humidity (ERH). For the example in Fig.2, the ERH is $12.5 \%$. Abrupt changes in the BLS peak positions/intensities must arise from abrupt changes in the particle's properties (shape, size, and refractive index). The fact that we can no longer properly fit the BLS spectra at $t_{E}$ with a Mie model further hints to the loss of the perfectly spherical shape of the particle. The difficulties with Mie fitting in the time window between $t_{E}$ to $t_{D, f}$ are indicated in panels c-d by larger error bars or missing data. We use the last BLS spectrum recorded just before $t_{E}$ where Mie fitting still provides good results to retrieve what we refer to as "particle efflorescence onset properties" ( $R$ and $m(\boldsymbol{\lambda})$ ). The time resolution in Fig. 2 is insufficient to unravel the dynamics during the efflorescence (i. e. in the time window $t_{E}$ to $\left.t_{D, i}\right)$. Details of these dynamics are addressed in section 3.2 based on BLS spectra recorded with high time resolution (10 $\mathrm{ms})$.

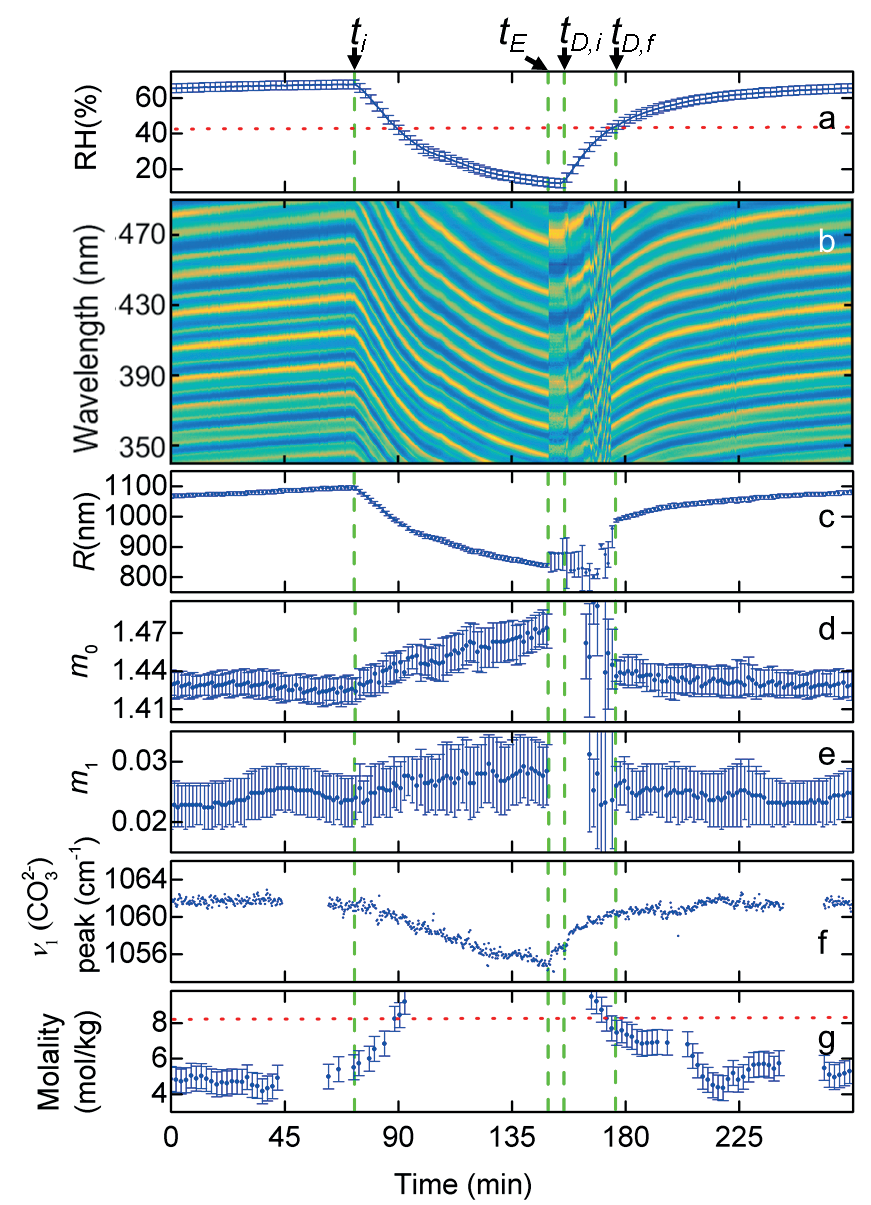

Fig. 2 Dehydration-hydration cycle of a single $\mathrm{K}_{2} \mathrm{CO}_{3}$ aerosol particle. See text for explanations of the green dashed lines and times $t_{i}, t_{E}, t_{D, i}, t_{D, f}$. a) Temporal evolution of the $\mathrm{RH}$. The red dotted line indicates the saturation $\mathrm{RH}$ of the bulk solution. b) Color-coded intensities in the BLS spectra as a function of wavelength and time. Yellow, green and blue colours indicate high, medium and low scattering intensity respectively. c) Temporal evolution of the particle radius $(R)$. d) Temporal evolution of the refractive index $m_{0}$ at $400 \mathrm{~nm}$ (non-dispersive part). e) Temporal evolution of the dispersive part $m_{1}$. The error bars in panels c-e indicate $95 \%$ credible intervals. f) Temporal evolution of the $\mathrm{CO}_{3}^{2-}$ band position. g) Temporal evolution of the molality of the particle. The red dotted line indicates the saturation molality of the bulk solution. The error bars in panel $\mathrm{g}$ are determined from the spread in the $\mathrm{CO}_{3}^{2-}$ band positions.

Re-humidification starting at $t_{D, i}$ is accompanied by multiple complex changes of the BLS peak positions/intensities until the particles becomes again spherical (liquid) at $t_{D, f}$. The $\mathrm{RH}$ at $t_{D, f}$ is the deliquescence relative humidity (DRH), which typically spreads over the range from $44 \%$ to $50 \% \mathrm{RH}$ in our measure- 
ments. Details of the kinetics during the deliquescence (i. e. in the time window $t_{D, i}$ to $t_{D, f}$ ) will be discussed in section 3.4. After time $t_{D, f}$, the particle behaves as an hygroscopic droplet which takes up water from the environment and thus increases in size. In this region where proper Mie fitting is again possible, the peak positions in the BLS spectra drift continuously to longer wavelengths.

\subsection{Multiple processes during efflorescence}

The phase transition dynamics during the efflorescence of single particles was studied with BLS with a time resolution of $10 \mathrm{~ms}$. All individual particles investigated experienced identical trapping and drying conditions (see section 2). A series of single particle studies revealed that at least two different types of efflorescence pathways can occur under otherwise identical conditions. The first pathway consists of a single process that is complete within the timescale of our resolution of $10 \mathrm{~ms}$. It is hereafter referred to as "prompt efflorescence". The second pathway consists of multiple successive processes that occur until the efflorescence is finalized. This pathway is referred to as "multistep efflorescence". The total time of multistep efflorescence processes can vary by 3 orders of magnitude between different particles from only a few $10 \mathrm{~ms}$ to more than 10 seconds.

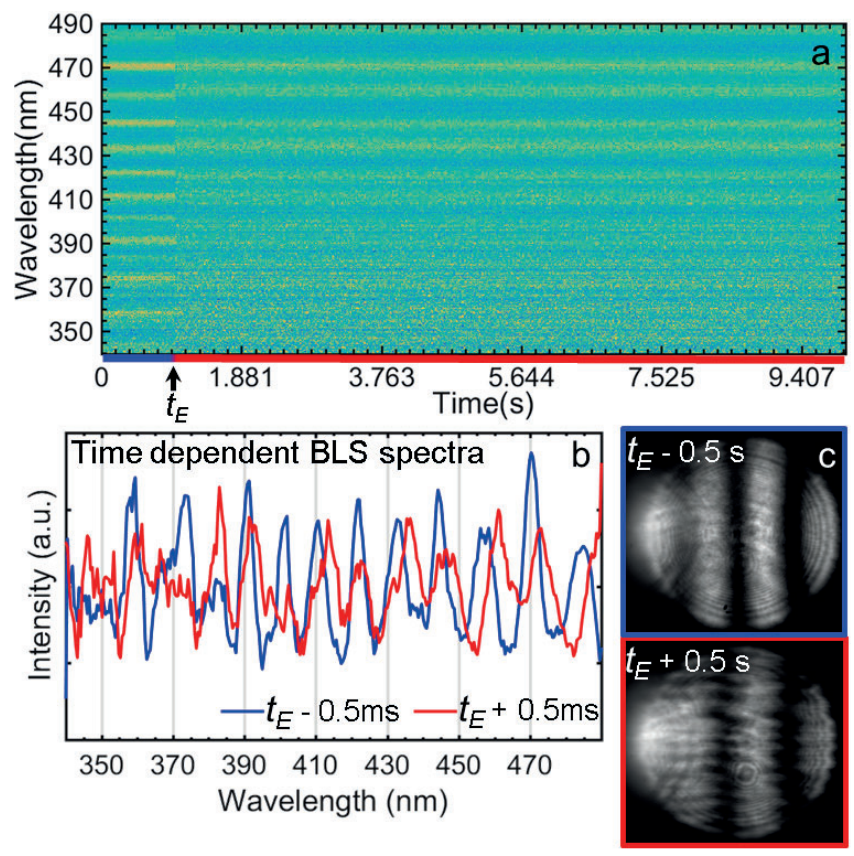

Fig. 3 Prompt (faster than 10ms) efflorescence of a submicron, aqueous $\mathrm{K}_{2} \mathrm{CO}_{3}$ particle $(R=954[882.5,969.5] \mathrm{nm})$. a) Color-coded intensities in the BLS spectra as a function of wavelength and time. Yellow, green and blue colours indicate high, medium and low scattering intensity respectively. b) BLS spectra recorded before $\left(t_{E}-0.5 \mathrm{~ms}\right.$; blue) and after $\left(t_{E}+\right.$ $0.5 \mathrm{~ms}$, black) efflorescence. c) TOAS patterns recorded before $\left(t_{E}-0.5\right.$ $\mathrm{s}$; upper) and after $\left(t_{E}+0.5 \mathrm{~s}\right.$, lower) efflorescence.

Fig. 3 shows a typical example for prompt efflorescence for a particle with an efflorescence onset radius of $R=954$ [882.5, $969.5] \mathrm{nm}$. (The numbers in the square brackets indicate the 95 $\%$ credible interval.) The wavelength- and time-dependent colour map of the BLS intensities (panel a) exhibits a single abrupt change at $t_{E}$. No further changes in the BLS spectra were observed before or after (measurement continued for at least 30 minutes after the efflorescence) this fast efflorescence process under constant RH conditions. The ERH for this particular example is 10.9 $\%$. For illustration; panel b shows two example BLS spectra that were recorded before $\left(t_{E}-0.5 \mathrm{~ms}\right)$ and after $\left(t_{E}+0.5 \mathrm{~ms}\right)$ the efflorescence event at $t_{E}$. The two spectra clearly differ from each other. The BLS results reveal that efflorescence in this system can happen faster than within $10 \mathrm{~ms}$. As mentioned in section 2, TAOS is usually not fast and characteristic enough to unambiguously probe phase transitions of submicron particles. Nevertheless, we have recorded TAOS spectra at lower time resolution together with BLS spectra. Two example TAOS patterns recorded before $\left(t_{E}-0.5 \mathrm{~s}\right)$ and after $\left(t_{E}+0.5 \mathrm{~s}\right)$ the transition are provided in panel c. The regular fringe pattern before efflorescence at $t_{E}-$ $0.5 \mathrm{~s}$ is consistent with a spherical, liquid particle. The pattern after efflorescence looks less regular, which could hint at deviation of the particle shape from a sphere in the solid state. Typically the scattering by a non-spherical particle is not azimuthally symmetric which causes the irregular pattern. However, disturbances in the TAOS patterns can also arise from the particle motion in the trap. Especially within the time resolution of our TAOS measurements fast particle motion could cause blurred images.

A typical example for multistep efflorescence is shown in Fig.4. The number of the different processes that occur during efflorescence, their characteristics (abrupt changes, slow drifts) and the total time the whole transition takes (between a few $10 \mathrm{~ms}$ and more than $10 \mathrm{~s}$ ) vary from particle to particle. For the example in Fig.4, the phase transition starts at the onset time $t_{E, i}=1.06 \mathrm{~s}$ and finalizes at $t_{E, f}=13.3 \mathrm{~s}$. The RH stays at a constant value of $12 \%$ during the whole process. Hence, the ERH for this particular example is $12 \%$. The BLS spectra in Fig.4a summarize five distinct time windows with characteristic temporal behavior:

- The BLS spectra do not exhibit any changes in the time window before the onset of efflorescence at $t_{E, i}$, where the BLS spectra change abruptly. In this time window, the particle consist of a supersaturated liquid, which is consistent with the regular fringe pattern observed in the TAOS in panel $c$ at $t=0 \mathrm{~s}$. A typical BLS spectrum recorded at the time indicated by the blue dashed line in trace a is shown as a blue line in trace $\mathrm{b}$. The efflorescence onset radius determined just before the onset of efflorescence is $R=2160$ [2150, 2220] $\mathrm{nm}$.

- Between $t_{E, i}$ and $t_{E, 1}$, all peaks in the BLS spectra shift slightly to shorter wavelengths by approximately $2 \mathrm{~nm}$, while the peak intensities and width remain fairly similar. A typical BLS spectrum recorded at the time indicated by the red dashed line in trace a is shown in the red line in trace b. This behaviour suggests that the particle slightly shrinks over time.

- A second and a third abrupt change are observed at $t_{E, 1}=3.8$ $\mathrm{s}$ and $t_{E, 2}=4.4 \mathrm{~s}$, respectively. During the $0.6 \mathrm{~s}$ in between these two abrupt changes, the peaks in the BLS spectra be- 
come less structured. An example BLS spectrum recorded at the time indicated by the black dashed line in trace a is shown in the black line in trace $b$.

- At $t_{E, 2}$, the particle settles in an intermediate state that lasts for a few seconds until efflorescence is complete at $t_{E, f}$. The BLS spectra are fairly stable in this time widow. A typical BLS spectrum recorded at the time indicated by the green dashed line in trace a is shown in the green line in trace $b$.

- After time $t_{E, f}$, no further changes in the BLS spectra are observed as long as the $\mathrm{RH}$ is constant, hence the particle remains in this state. In this particular example the measurement continued at constant RH condition for 35 minutes after $t_{E, f}$ (data not shown). An example BLS spectrum at this final state is shown in the gray line in trace $b$.

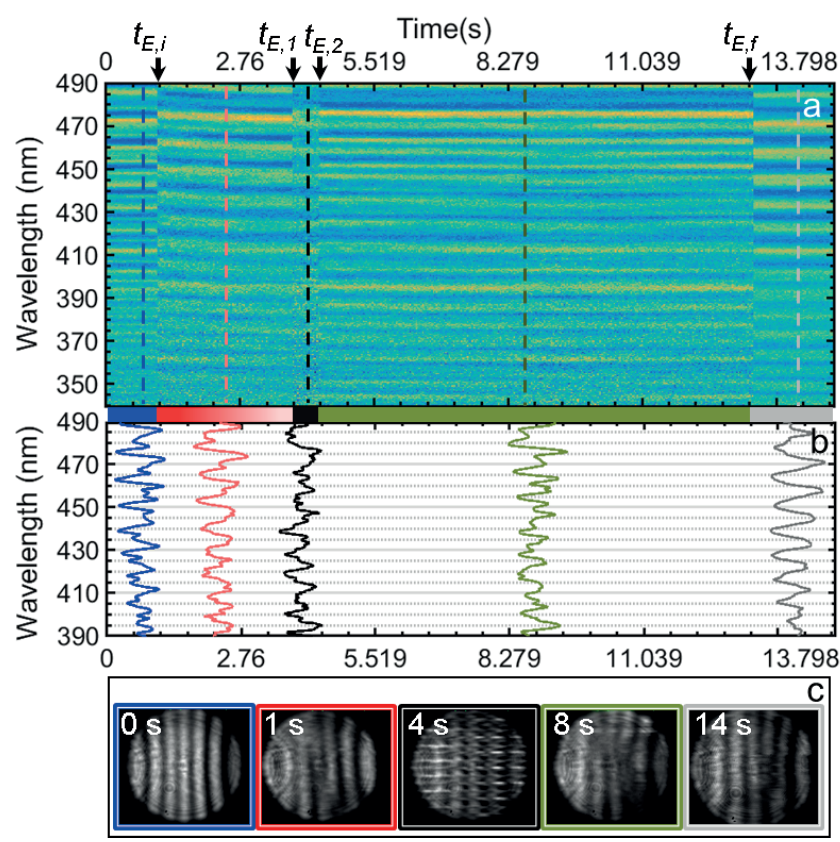

Fig. 4 Multistep efflorescence of a micron-sized aqueous $\mathrm{K}_{2} \mathrm{CO}_{3}$ particle $(R=2160[2150,2220] \mathrm{nm}) . t_{E, i}$ and $t_{E, f}$ indicates the onset and the end, respectively, of the efflorescence process. a) Color-coded intensities in the BLS spectra as a function of wavelength and time. Yellow, green and blue colours indicate high, medium and low scattering intensity respectively. The time resolution was $10 \mathrm{~ms}$. b) Example BLS spectra recorded at different times, which are indicated in panel a by the dashed lines. $\mathrm{c}$ ) Example TAOS recorded at different times. TAOS measurements were performed once per second. The RH was unchanged at $12 \%$ over the time window shown.

The TAOS in panel c recorded at different times after the onset of efflorescence at $t_{E, i}$ show less regular patterns compared with the one recorded at $t=0 \mathrm{~s}$; i. e. before the onset of efflorescence. As mentioned before these irregular patterns hint at deviations from perfect spherical particle shapes. With unknown shape, however, a further analysis of the spectra is not possible at this point. The comparison of TAOS and BLS spectra again reveals that BLS is superior when it comes to the detection of phase transitions because it is much faster and more characteristic. The complexity of the whole process with multiple intermediate steps is only uncovered in the BLS spectra.

The significant differences between BLS spectra recorded within only $20 \mathrm{~ms}$ around the onset of efflorescence is visualized in Fig. 5 for the example of a submicron particle $(R=626.9$ [617.0 $633.5] \mathrm{nm})$. Blue, black, and red show spectra at $t_{E, i^{-}} 10 \mathrm{~ms}, t_{E, i}$, and $t_{E, i}+10 \mathrm{~ms}$, respectively. This suggests significant differences in the particle properties (i.e. particle phase, shape) at $t_{E, i}-10$ $\mathrm{ms}, t_{E, i}$, and $t_{E, i}, t_{E, i}+10 \mathrm{~ms}$. Hence, at the onset of efflorescence $t_{E, i}$ the particle is in an intermediate state that is different from both the previous supersaturated liquid state and the subsequent state. It is thus clear that the phase transition exemplified by Fig. 5 is a very fast multi-step rather than a prompt process. Continuous changes in the particle properties (e.g. its size) would not produce the pronounced spectral changes observed in Fig.5. This finding could only be revealed by the high sensitivity and time resolution of our BLS measurements. The fact the TAOS shown in Fig.5 just before and after $t_{E, i}$ are hardly distinguishable illustrates the limitations of TAOS in detecting very fast multi-step processes.

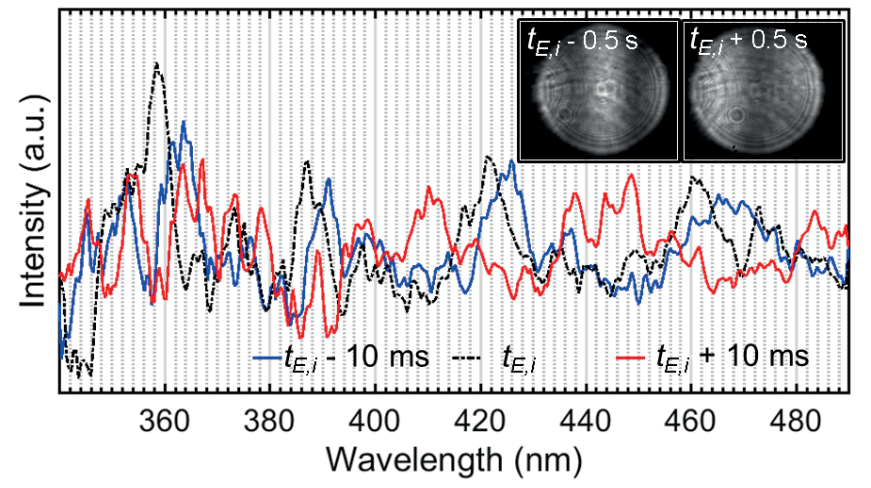

Fig. 5 BLS spectra recorded at $t_{E, i^{-}} 10 \mathrm{~ms}$ (blue), $t_{E, i}$ (black), and $t_{E, i}+$ $10 \mathrm{~ms}$ (red) for a submicron $\mathrm{K}_{2} \mathrm{CO}_{3}$ particle $(R=626.9$ [617.0 633.5] nm). Inset: TAOS patterns before $t_{E, i^{-}} 0.5 \mathrm{~s}$ and after $t_{E, i}+0.5 \mathrm{~s}$ the onset of efflorescence.

Typical exposure times between 1 s to 60 s required for Raman spectroscopy made Raman observation of fast processes during efflorescence $\left(t_{E, i}\right.$ and $\left.t_{E, f}\right)$ in general impossible, so that we focused the Raman studies on the analysis of particles before and after the efflorescence during which no changes were observed. We observed very similar Raman spectra for all particles investigated. As the efflorescence pathways differ between particles, this suggests that the final state of the effloresced particles is independent of the efflorescence pathway. Typical Raman spectra before and after the efflorescence are shown in Fig.6 in the region of the $v_{3}(\mathrm{OH})$ and the $v_{1}\left(\mathrm{CO}_{3}^{2-}\right)$ band $(R=954$ [883 970] nm). In order to compare our single particle efflorescence measurements with crystalline samples we measured the Raman shift of solid powder samples (Anhydrate: Acros, $\geq 99.0 \%$ and sesquihydrate: SigmaAldrich, $\geq 99.995 \%$ ). The supersatured liquid state before efflorescence is characterized by a broad unstructured $v_{3}(\mathrm{OH})$ band with a maximum at $3300 \mathrm{~cm}^{-1}$. After efflorescence, the $v_{3}(\mathrm{OH})$ band does not vanish but it becomes more structured and its maximum shifts to $3185 \mathrm{~cm}^{-1}$. This spectrum compares well with 
the sesquihydrate solid powder (see Fig.6) suggesting the formation of a crystalline $\mathrm{K}_{2} \mathrm{CO}_{3}$ particle with remaining water content. This is also supported by the position of the $v_{1}\left(\mathrm{CO}_{3}^{2-}\right)$ band, which after effloresence $\left(1060 \mathrm{~cm}^{-1}\right)$ lies closer to the band position of the crystalline powders $\left(1060.5 \mathrm{~cm}^{-1}\right)$ than to the position of the liquid before efflorescence $\left(1056.0 \mathrm{~cm}^{-1}\right)$. In addition, the $v_{1}\left(\mathrm{CO}_{3}^{2-}\right)$ band widths of the effloresced particle is much narrower than for the liquid particle $\left(\mathrm{FWHM}^{*}\right.$ of $16.9 \mathrm{~cm}^{-1}$ and $23.3 \mathrm{~cm}^{-1}$, respectively). However, the FWHM after efflorescence still remains $\sim 2$ times larger than for the solid powder samples. This difference shows that the effloresced particle does not form the same crystal structure as the powder. The peak area analysis is done by determining the ratios of the areas of $v_{3}(\mathrm{OH})$ and $v_{1}\left(\mathrm{CO}_{3}^{2-}\right)$ bands. This ratio is $\sim 25 \%$ smaller for the effloresced particle than the sesquihydrate. Although this result is suggesting a lower water content in the particle after efflorescence than in the sesquihydrate, quantitative analysis is not possible for lack of experimental data for the Raman scattering cross-sections of crystalline $\mathrm{K}_{2} \mathrm{CO}_{3}$.
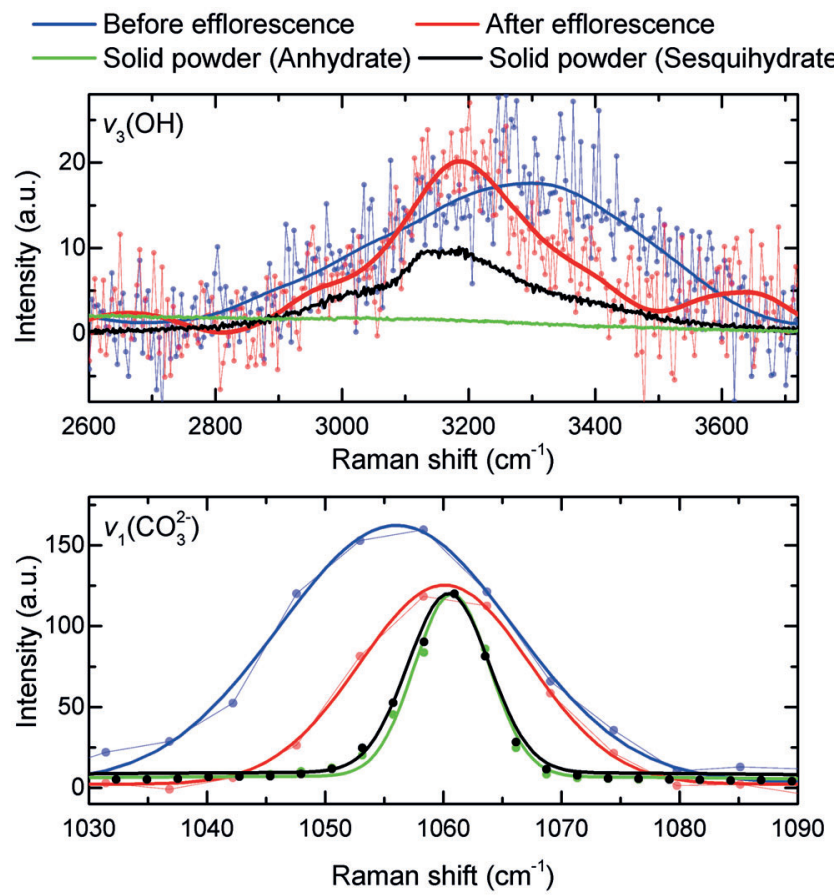

Fig. 6 Single particle Raman spectra in the region of the $v_{3}(\mathrm{OH})$ and the $v_{1}\left(\mathrm{CO}_{3}^{2-}\right)$ band. Blue and red lines show single particle measurements of a particle in the supersaturated liquid state before efflorescence at $\mathrm{ERH}=10.94 \%$ and in the solid state after efflorescence, respectively. Anhydrate and sesquihydrate powders of $\mathrm{K}_{2} \mathrm{CO}_{3}$ are measured with the same spectrometer as the single particle measurements. See the text for details. The points represent the raw data. The full lines correspond to smoothed raw data

\subsection{Efflorescence relative humidity}

From efflorescence studies of 22 individual particles, we have determined the ERH and the particle efflorescence onset radius $R$.

* FWHM: Full width at half maximum
We observed a wide range of ERHs from $9 \%$ to $25 \%$ with typical absolute errors of $2 \%$ (Fig.7). Fig.7 shows a tendency of large particles to effloresce at lower ERH, while for small particles the ERH varies over the whole range. The different measurement points in Fig.7 are classified according to the type of efflorescence transition (see section 3.2): prompt, multistep, and $\mathrm{n} / \mathrm{a}$. n/a referres to BLS experiments that were performed with lower time resolution ( $>10 \mathrm{~ms}$ ) for which it was not possible to distinguish between prompt and multistep. Fig.7 suggests that particles larger than $\sim 2 \mu \mathrm{m}$ seem to have a preference for multistep transition. Prompt transition were only observed for particles with radii smaller than $\sim 2 \mu \mathrm{m}$. However, the limited number of measurements is insufficient to decide whether these are robust trends.

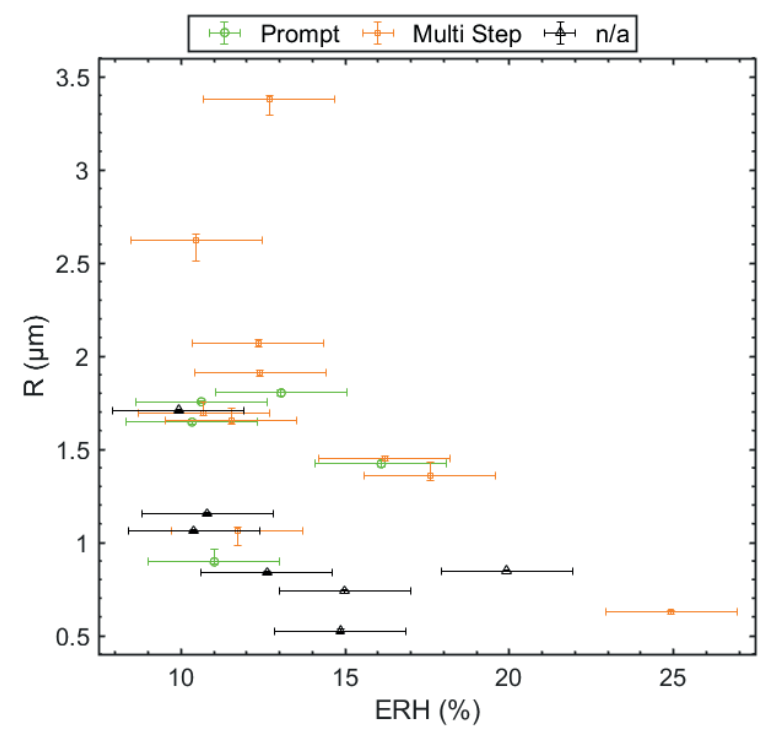

Fig. 7 Efflorescence onset radius $R$ as a function of the ERH retrieved from 22 single particle efflorescence experiments. The error bars indicate $95 \%$ credible intervals for the absolute error.

\subsection{Multiple processes during deliquescence and DRH}

We have studied the deliquescence of particles with different initial sizes $(600-4000 \mathrm{~nm})$ over the course of several dehydrationhydration cycles. Even though each particle and cycle showed a unique dynamical behavior the general trend was similar. This trend is illustrated by the example in Fig.8, which shows the BLS spectra and the evolution of the RH over the course of a deliquescence experiment starting at $t_{D, i}$. During the initial increase of the $\mathrm{RH}$ from $9.5 \%$ to $25-35 \%$ (30 \% in Fig.8) the peak positions in the BLS spectra shift slightly to longer wavelengths. This behaviour suggests a small growth of the particle size that could arise from an initial water uptake on the effloresced particle, possibly from the formation of a liquid layer coating the particle, as previously observed $7,30,31,33-36,53$. This initial shift of the BLS peaks in Fig. 8 is $\sim 2.5 \mathrm{~nm}$ (e.g. 454.3 to $456.8 \mathrm{~nm}$ ). Assuming that only the size of the particle is changing, such a shift corresponds to an increase of the particle radius by $\sim 10 \mathrm{~nm}$ (see ${ }^{\dagger}$ for details). At an RH of 25-35\% (30\% in Fig.8), a series 
of multiple processes starts and continues until the particle completely dissolves at $t_{D, f}$. The multiple processes include abrupt changes (faster than $10 \mathrm{~ms}$ ) and regions of continuous shifts in the peak positions/intensities in the BLS spectra, suggesting corresponding abrupt and more continuous changes, respectively, in the particle properties. After time $t_{D, f}$, the liquid particle undergoes hygroscopic growth (see also Fig.2). The deliquescence relative humidity DRH (determined at $t_{D, f}$ ) varies between 44-50 $\%$. The differences between the individual effloresced particles just before the start of the hydration cycles certainly explain part of the unique dynamical behaviour observed for each individual particle and cycle. Other phenomena not related to differences in the initial state could also contribute. However, at the current stage we are not able to further specify these phenomena. It has previously been observed that initial state properties such as the particle morphology and the roughness or the porosity of the particle surface can determine the amount of water vapor the surface can adsorb as well as the remaining water content in the particle. 7,30,31,33-36,53,54 Several of these references also report the initial formation of a few nm thick water layer, which seems consistent with our observation (slight initial shift of the peak positions in the BLS spectra to longer wavelengths in Fig.8). Such initial coatings can alter the particle morphology, e.g. by rounding corners and removing roughness. An important question for aerosol studies concerns the effect of the particle size, i.e. its radius of curvature, on the water uptake, which in turn influences the DRH of submicron particles. ${ }^{1,55}$ In the present study, we did not observe a systematic dependence of the DRH on the particle size. We do, however, observe a pronounced variation of typically $40 \%$ in the total BLS intensity, in the time window between $t_{D, i}$ and $t_{D, f}$. The time evolution of the total BLS intensity was determined by integrating the BLS spectra over the wavelength range $320 \mathrm{~nm} \leq \lambda \leq 490 \mathrm{~nm}$ at each time step. Hence, initial water uptake and accompanying complex deliquescence processes might be important for accurate estimations of radiative transfer in the atmospheric studies. ${ }^{56}$

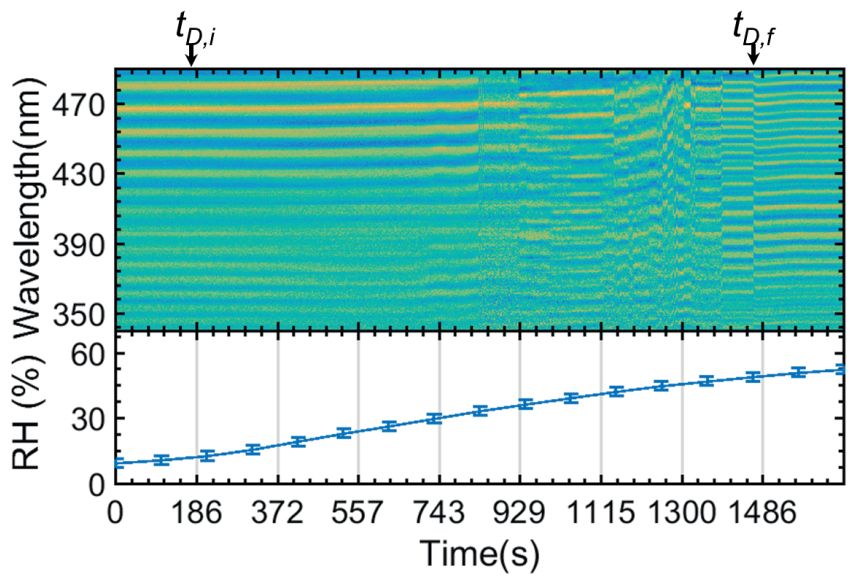

Fig. 8 Top: Color-coded intensities in the BLS spectra as a function of wavelength and time during efflorescence of a single optically trapped particle $(R=2070.5[2052,2086] \mathrm{nm})$. Bottom: Temporal evolution of the $\mathrm{RH}$. For the sake of clarity only a few error bars are shown.

\section{Summary}

We have investigated the hygroscopic behaviour of single, optically trapped $\mathrm{K}_{2} \mathrm{CO}_{3}$ particles using broadband light scattering (BLS) and Raman spectroscopy. The occurrence of complex multiple processes during efflorescence and deliquescence cycles has previously been reported for deposited particles, or single, isolated particles with sizes in the upper micrometer range. The major goal of the present work was to clarify whether such complex multiple processes also occur in unsupported, submicron sized particles. The isolation of single particles in air avoids issues with the interpretation of multiple processes arising from ensemble averaging and from heterogeneous processes involving the supporter. BLS allowed us to probe the dynamics with high time resolution down to $10 \mathrm{~ms}$. The major observations can be summarized as follows:

- Efflorescence and deliquescence are both accompanied by complex multiple processes, which correspond to either abrupt (shorter than $10 \mathrm{~ms}$ ) or continuous changes in the particle properties.

- Two different types of efflorescence pathways occur under identical conditions: (i)"Prompt efflorescence" consisting of a single process which is complete within $10 \mathrm{~ms}$. (ii) "Multistep efflorescence" consisting of multiple successive processes that can take up to several seconds in total. The Raman spectra of the effloresced particles are identical and are consistent with crystalline hydrates.

- Deliquescence begins with an initial uptake of water, possibly the formation of a liquid layer coating the particle, and continuous with a series of multiple steps until the DRH is reached where the particle liquefies completely.

These observations clearly demonstrate the evidence of complex multiple processes during efflorescence and deliquescence also in unsupported, submicron sized particles. BLS is fast and very sensitive to small variations in particle size $(\sim \mathrm{nm})$, refractive index $\left(<10^{-3}\right)$ and morphology and thus fulfills many prerequisites for the detection of multiple fast processes. The combination of single particle trapping and BLS is also a very attractive method to retrieve the frequency dependent refractive index of highly supersaturated solutions, which is difficult to obtain from other studies. The only caveat is that BLS spectra alone can generally not provide a meaningful quantification of particle size and refractive index for non-spherical particles; i. e. in the region from the onset of efflorescence to the completion of deliquescence. Additional knowledge of the particle shape would be very valuable here. We have very recently demonstrated that the missing shape information can be retrieved by holographic imaging of single, optically-trapped submicron sized particles with a resolution of 240 microseconds (manuscript is in preparation). We hope that the planned combination of BLS with the new holographic imaging method will open up a new way for a better quantification of particle properties during efflorescence and deliquescence. 


\section{Acknowledgment}

We are very grateful to David Stapfer and Markus Steger from the ETH Zürich Laboratory of Physical Chemistry shops for their assistance in developing the experimental setup. This work was supported by the Swiss National Science Foundation (SNSF grant no. 200020_172472) and ETH Zürich.

\section{References}

1 Y. Cheng, H. Su, T. Koop, E. Mikhailov and U. Pöschl, Nat. Commun., 2015, 6, 5923.

2 G. C. Roberts and A. Nenes, Aerosol Sci. Technol., 2005, 39, 206-221.

3 O. Boucher, D. Randall, P. Artaxo, C. Bretherton, G. Feingold, P. Forster, V.-M. Kerminen, Y. Kondo, H. Liao, U. Lohmann, P. Rasch, S. K. Satheesh, S. Sherwood, B. Stevens and X. Y. Zhang, IPCC, 2013: Climate Change 2013: The Physical Science Basis. Contribution of Working Group I to the Fifth Assessment Report of the Intergovernmental Panel on Climate Change, Cambridge University Press, Cambridge, United Kingdom and New York, NY, USA, 2013.

4 D. L. Nuding, E. G. Rivera-Valentin, R. D. Davis, R. V. Gough, V. F. Chevrier and M. A. Tolbert, Icarus, 2014, 243, 420-428.

5 L. J. Mauer and L. S. Taylor, Annu. Rev. Food Sci. Technol., 2010, 1, 41-63.

6 A. Baldelli, R. M. Power, R. E. H. Miles, J. P. Reid and R. Vehring, Aerosol Sci. Technol., 2016, 50, 693-704.

7 A. D. Estillore, H. S. Morris, V. W. Or, H. D. Lee, M. R. Alves, M. A. Marciano, O. Laskina, Z. Qin, A. V. Tivanski and V. H. Grassian, Phys. Chem. Chem. Phys., 2017, 19, 21101-21111.

8 G. Biskos, D. Paulsen, L. M. Russell, P. R. Buseck and S. T. Martin, Atmos. Chem. Phys., 2006, 6, 4633-4642.

9 S. T. Martin, Chem. Rev., 2000, 100, 3403-3453.

10 M. Shiraiwa, Y. Li, A. P. Tsimpidi, V. A. Karydis, T. Berkemeier, S. N. Pandis, J. Lelieveld, T. Koop and U. Pöschl, Nat. Commun., 2017, 8, 15002.

11 S. Karthika, T. K. Radhakrishnan and P. Kalaichelvi, Cryst. Growth Des., 2016, 16, 6663-6681.

12 R. P. Sear, Europhys. Lett., 2008, 83, 66002.

13 A. K. Shchekin, I. V. Shabaev and O. Hellmuth, J. Chem. Phys., 2013, 138, 054704.

14 A. V. Mokshin and B. N. Galimzyanov, Phys. Chem. Chem. Phys., 2017, 19, 11340-11353.

15 J. Baumgartner, A. Dey, P. H. H. Bomans, C. Le Coadou, P. Fratzl, N. A. J. M. Sommerdijk and D. Faivre, Nat. Mater., 2013, 12, 310-314.

16 A. F. Wallace, L. O. Hedges, A. Fernandez-Martinez, P. Raiteri, J. D. Gale, G. A. Waychunas, S. Whitelam, J. F. Banfield and J. J. De Yoreo, Science, 2013, 341, 885-889.

17 D. Chakraborty and G. N. Patey, Chem. Phys. Lett., 2013, 587, 25-29.

18 D. Gebauer, M. Kellermeier, J. D. Gale, L. Bergström and H. Cölfen, Chem. Soc. Rev., 2014, 43, 2348-2371.

19 N. D. Loh, S. Sen, M. Bosman, S. F. Tan, J. Zhong, C. A. Nijhuis, P. Král, P. Matsudaira and U. Mirsaidov, Nat. Chem.,
$2017,9,77-82$.

20 Y. Djikaev and E. Ruckenstein, J. Chem. Phys., 2006, 124, 124521.

21 P. G. Vekilov, Nanoscale, 2010, 2, 2346-2357.

22 M. Iwamatsu, J. Chem. Phys., 2011, 134, 164508.

23 G. C. Sosso, J. Chen, S. J. Cox, M. Fitzner, P. Pedevilla, A. Zen and A. Michaelides, Chem. Rev., 2016, 116, 7078-7116.

24 J. F. Lutsko, New Perspectives on Mineral Nucleation and Growth, Springer, Cham, 2017, pp. 25-41.

25 A. A. Barsuk and F. Paladi, Physica A: Statistical Mechanics and its Applications, 2017, 487, 74-92.

26 D. Zahn, Chemphyschem, 2015, 16, 2069-2075.

27 M. H. Nielsen, S. Aloni and J. J. De Yoreo, Science, 2014, 345, 1158-1162.

28 S. Lee, H. S. Wi, W. Jo, Y. C. Cho, H. H. Lee, S.-Y. Jeong, Y.-I. Kim and G. W. Lee, Proc. Natl. Acad. Sci. U. S. A., 2016, 113, 13618-13623.

29 P. Mirabel, H. Reiss and R. K. Bowles, J. Chem. Phys., 2000, 113, 8194-8199.

30 E. J. Freney, S. T. Martin and P. R. Buseck, Aerosol Sci. Technol., 2009, 43, 799-807.

31 M. E. Wise, S. T. Martin, L. M. Russell and P. R. Buseck, Aerosol Sci. Technol., 2008, 42, 281-294.

32 L. Krämer, U. Pöschl and R. Niessner, J. Aerosol Sci., 2000, 31, 673-685.

33 E. Mikhailov, S. Vlasenko, R. Niessner and U. Pöschl, Atmos. Chem. Phys., 2004, 4, 323-350.

34 V. Zelenay, M. Ammann, A. Křepelová, M. Birrer, G. Tzvetkov, M. G. C. Vernooij, J. Raabe and T. Huthwelker, J. Aerosol Sci., 2011, 42, 38-51.

35 D. A. Bruzewicz, A. Checco, B. M. Ocko, E. R. Lewis, R. L. McGraw and S. E. Schwartz, J. Chem. Phys., 2011, 134, 044702.

36 T.-C. Hsiao, L.-H. Young, Y.-C. Tai and K.-C. Chen, Aerosol Sci. Technol., 2016, 50, 568-577.

37 H. Vortisch, B. Krämer, I. Weidinger, L. Wöste, T. Leisner, M. Schwell, H. Baumgärtel and E. Rühl, Phys. Chem. Chem. Phys., 2000, 2, 1407-1413.

38 C. Braun and U. Krieger, Opt. Express, 2001, 8, 314-321.

39 J. W. Lu, M. Isenor, E. Chasovskikh, D. Stapfer and R. Signorell, Rev. Sci. Instrum., 2014, 85, 095107.

40 S. H. Jones, M. D. King and A. D. Ward, Phys. Chem. Chem. Phys., 2013, 15, 20735-20741.

41 G. David, K. Esat, I. Ritsch and R. Signorell, Phys. Chem. Chem. Phys., 2016, 18, 5477-5485.

42 A. A. Zardini, U. K. Krieger and C. Marcolli, Opt. Express, 2006, 14, 6951-6962.

43 J. W. Cremer, K. M. Thaler, C. Haisch and R. Signorell, Nat. Commun., 2016, 7, 10941.

44 L. Greenspan, J. Res. Natl. Bur. Stan. Sect. A., 1977, 81A, 89.

45 D. I. Strokotov, M. A. Yurkin, K. V. Gilev, D. R. van Bockstaele, A. G. Hoekstra, N. B. Rubtsov and V. P. Maltsev, J. Biomed. Opt., 2009, 14, 064036.

46 M. I. Mishchenko, L. D. Travis and D. W. Mackowski, J. Quant. 
Spectrosc. Radiat. Transf., 1996, 55, 535-575.

47 M. I. Mishchenko and M. A. Yurkin, Opt. Lett., 2017, 42, 494497.

48 R. Salter, J. Chu and M. Hippler, Analyst, 2012, 137, 46694676.

49 J. D. Frantz, Chem. Geol., 1998, 152, 211-225.

50 W. W. Rudolph, G. Irmer and E. Königsberger, Dalton Trans., 2008, 900-908.

51 Q. Sun and C. Qin, Chem. Geol., 2011, 283, 274-278.
52 T. E. Acosta-Maeda, A. K. Misra, J. N. Porter, D. E. Bates and S. K. Sharma, Appl. Spectrosc., 2017, 71, 1025-1038.

53 E. Mikhailov, S. Vlasenko, S. T. Martin, T. Koop and U. Pöschl, Atmos. Chem. Phys., 2009, 9, 9491-9522.

54 E. Ruckenstein and G. O. Berim, J. Colloid Interface Sci., 2010, 351, 277-282.

55 A. P. Ault and J. L. Axson, Anal. Chem., 2017, 89, 430-452.

56 M. C. Yeung and C. K. Chan, Aerosol Sci. Technol., 2010, 44, 269-280. 